\title{
ON UNIFORMLY DISTRIBUTED SEQUENCES OF INTEGERS AND POINCARÉ RECURRENCE III
}

\author{
R. NAIR
}

Let $S$ be a semigroup contained in a locally compact Abelian group $G$. Let $\widehat{G}$ denote the Bohr compactification of $G$. We say that a sequence $\mathbf{k}=\left(k_{n}\right)_{n=1}^{\infty}$ contained in $S$ is Hartman uniform distributed on $G$ if

$$
\lim _{N \rightarrow \infty} \frac{1}{N} \sum_{n=1}^{N} \chi\left(k_{n}\right)=0
$$

for any character $\chi$ in $\widehat{G}$. Suppose that $\left(T_{g}\right)_{g \in S}$ is a semigroup of measurable measure preserving transformations of a probability space $(X, \beta, \mu)$ and $B$ is an element of the $\sigma$-algebra $\beta$ of positive $\mu$ measure. For a map $T: X \rightarrow X$ and a set $A \subseteq X$ let $T^{-1} A$ denote $\{x \in X: T x \in A\}$. In an earlier paper, the author showed that if $\mathbf{k}$ is Hartman uniform distributed then

$$
\lim _{M \rightarrow \infty} \frac{1}{M} \sum_{n=1}^{M} \mu\left(B \cap\left(T_{k_{n}}\right)^{-1} B\right) \geqslant \mu(B)^{2} .
$$

In this paper we show that $\geqslant$ cannot be replaced by $=$. A more detailed discussion of this situation ensues.

Let $G$ be a locally compact Abelian group and let $\mathbf{k}=\left(k_{n}\right)_{n=1}^{\infty}$ be a sequence contained in a semigroup $S$ contained in $G$. We say that $\mathbf{k}=\left(k_{n}\right)_{n=1}^{\infty}$ is Hartman uniform distributed if for each non-trivial character $\chi$ in the dual group $\hat{G}$ of $G$ we have

$$
\lim _{N \rightarrow \infty} \frac{1}{N} \sum_{n=1}^{N} \chi\left(k_{n}\right)=0 .
$$

For a set $X$ let $\beta$ denote a $\sigma$-algebra of its subsets and let $\mu$ be a probability measure defined on them. We say that a measurable map $T$ from $X$ to itself is measure preserving if for any element $A$ of $\beta$, denoting by $T^{-1} A$ the set $\{x \in X: T x \in A\}$, we have $\mu\left(T^{-1} A\right)=\mu(A)$ for all $A$ in $\beta$. In [1] the following is shown.

Received 29th April, 2003

Copyright Clearance Centre, Inc. Serial-fee code: 0004-9727/03 \$A2.00+0.00. 
TheOREM A. Suppose that $\mathbf{k}=\left(k_{j}\right)_{j=1}^{\infty}$ is Hartman uniformly distributed on a locally compact Abelian group $G$, containing a semigroup $S$ containing k. Suppose that $\left(T_{g}\right)_{g \in S}$ is a semigroup of measurable measure preserving transformations of a probability space $(X, \beta, \mu)$ and that $B$ is an element of $\beta$ of positive $\mu$ measure. Then

$$
\lim _{M \rightarrow \infty} \frac{1}{M} \sum_{n=1}^{M} \mu\left(B \cap\left(T_{k_{n}}\right)^{-1} B\right) \geqslant \mu(B)^{2} .
$$

The existence of the limit is part of the conclusion to Theorem A. We however have the following theorem.

THEOREM B. The $\geqslant$ in the statement of Theorem $A$ can't be replaced by $=$.

PROof: Suppose otherwise and we shall specialise to the case $G=\mathbf{Z}$. Recall that a sequence of real numbers $\left(x_{n}\right)_{n=1}^{\infty}$ is said to be uniformly distributed modulo one, if for each interval $I$ that is closed on the left and open on the right we have

$$
\lim _{N \rightarrow \infty} \frac{1}{N} \sum_{n=1}^{N} \chi_{I}\left(\left\{x_{n}\right\}\right)=|I|
$$

Here $\{x\}$ denotes the fractional part of the real number $x, \chi_{I}$ denotes the characteristic function of the interval $I$ and $|I|$ denotes its Lebesgue measure. As no ambiguity should arise, for a finite set $F$ we denote its cardinality also by $|F|$. We say that a sequence of natural numbers $\mathbf{k}=\left(k_{j}\right)_{j=1}^{\infty}$ is uniformly distributed on $\mathbf{Z}$ if for each integer $m$ in $\mathbf{N}$ and each integer $l$ in $[0, m-1] \cap \mathbf{Z}$ we have

$$
\lim _{N \rightarrow \infty} \frac{1}{N}\left|\left\{j \in[0, N-1) \cap \mathbf{Z}: k_{j} \equiv l \bmod m\right\}\right|=\frac{1}{m} .
$$

In [1] it is shown that $\mathbf{k}=\left(k_{n}\right)_{n=1}^{\infty}$ is Hartman uniform distributed on $\mathbf{Z}$ if $\mathbf{k}$ is uniformly distributed on $\mathbf{Z}$ and for each irrational number $\alpha$, the sequence $\left(k_{n} \alpha\right)_{n=1}^{\infty}$ is uniformly distributed modulo one. Also in [1] it is shown that there are many sequences with this property. Suppose $(X, \beta, \mu)$ is any probability space, $T: X \rightarrow X$ is any measurable, measure preserving transformation of $X$ and $B$ is a $T$ invariant set in $\beta$ in the sense that $T^{-1} B=B$. Then for any sequence of natural numbers $\mathbf{k}=\left(k_{n}\right)_{n=1}^{\infty}$ and any natural number $M$

$$
\frac{1}{M} \sum_{n=1}^{M} \mu\left(B \cap T^{-k_{n}} B\right)=\mu(B),
$$

for each $M \geqslant 1$. Hence if

$$
\lim _{M \rightarrow \infty} \frac{1}{M} \sum_{n=1}^{M} \mu\left(B \cap\left(T^{k_{n}}\right)^{-1} B\right)=\mu(B)^{2} .
$$


then

$$
\mu(B)=\mu(B)^{2} .
$$

Therefore $\mu(B)$ is either zero or one. Now set $X=[0,1), \mu$ is equal to the Lebesgue measure, $T x=\langle x+1 / 2\rangle$ and $B=[0,(1 / 4)) \cup[(1 / 2),(3 / 4))$. Then $T$ preserves $\mu$ and $B$ is obviously $T$ invariant while $\mu(B)=1 / 2$. This is a contradiction.

Recall that a measurable, measure preserving map $T: X \rightarrow X$ of a probability space $(X, \beta, \mu)$ is ergodic if given any $B$ in $\beta$ with $T^{-1} B=B$ then $\mu(B)$ is either zero or one. Plainly our example above works because $T$ is not ergodic. It is unclear to the author whether Theorem A remains true with $\geqslant$ replaced by = under the additional assumption that $T$ is ergodic.

We need to establish some standard notation. We say that a statement is true $\mu$ almost everywhere if the subset of $X$ on which it is true has full $\mu$ measure. We also say that two functions $f$ and $g$ are equivalent if $f-g=0 \mu$ almost everywhere. Let $\|f\|=\left(\int_{X}|f|^{2} d \mu\right)^{1 / 2}$ and let $L^{2}=L^{2}(X, \beta, \mu)$ denote the space of equivalence classes for $\mu$ measurable functions such that the norm $\|f\|$ is finite. Given a sequence of functions $\left(f_{N}\right)_{N=1}^{\infty}$ defined on a probability space $(X, \beta, \mu)$ we say that $\left(f_{N}\right)_{N=1}^{\infty}$ converges to a function $g$ defined on $(X, \beta, \mu)$ in $L^{2}$ norm if $\lim _{N \rightarrow \infty}\left\|f_{N}-g\right\|=0$. We say that $\left(f_{N}\right)_{N=1}^{\infty}$ converges almost everywhere to $g$ if $\mu\left(\left\{x \in X: \lim _{N \rightarrow \infty} f_{N}(x)\right.\right.$ $=g(x)\})=1$. In [2] it is shown that if $\mathbf{k}=\left(k_{n}\right)_{n \geqslant 1}$ is Hartman uniformly distributed on $\mathbf{Z}$ then this is equivalent to the statement that if $f \in L^{2}(X, \beta, \mu)$ and $T: X \rightarrow X$ is measurable and measure preserving, then

$$
\lim _{N \rightarrow \infty}\left\|\frac{1}{N} \sum_{n=1}^{N} f\left(T^{k_{n}} x\right)-\mathbf{E}(f \mid \mathcal{I})\right\| .
$$

Here $\mathbf{E}(f \mid \mathcal{I})$ is the projection of $f$ onto the subspace of $L^{2}$ of $T$ invariant functions. Suppose that instead of being Hartman uniform distributed on $\mathbf{Z}$ the sequence $\mathbf{k}$ $=\left(k_{n}\right)_{n \geqslant 1}$ has the property that if $f \in L^{2}(X, \beta, \mu)$ and $T: X \rightarrow X$ is measurable and measure preserving then

$$
\lim _{N \rightarrow \infty} \frac{1}{N} \sum_{n=1}^{N} f\left(T^{k_{n}} x\right)=\mathbf{E}(f \mid \mathcal{I}),
$$

almost everywhere with respect to $\mu$. Note that hypothesis (2) says that if $f$ is in $L^{2}(X, \beta, \mu)$ then the sequence $\left(1 / N \sum_{n=1}^{N} f\left(T^{k_{n}} x\right)\right)_{N=1}^{\infty}$ converges to $\mathbf{E}(f \mid \mathcal{I})(x)$ in $L^{2}$ norm and (3) says the convergence is almost everwhere. In general convergence 
almost everywhere does not necessarily imply convergence in norm, nor vice-versa. The hypothesis (3) does however imply the hypothesis (2). See [4, Lemma 4] for a proof of this. Also, in the case where $G=\mathbf{Z}$, to prove (3) we need a much more refined condition on $\mathbf{k}$ than (1). See [3] for details of this. A number of families of sequences of natural numbers for which (3) is true can also be found in [3]. In [2] it is shown that in the presence of (3) the ergodicity of $T$ is equivalent to

$$
\lim _{M \rightarrow \infty} \frac{1}{M} \sum_{n=1}^{M} \mu\left(A \cap T^{-k_{n}} B\right)=\mu(B) \mu(A),
$$

for each pair $A, B \in \beta$. This of course implies (1) for the particular transformation $T$. In the absence of (3) condition (4) still implies the ergodicity of $T$. For ergodic $T$, property (1) for $\mathbf{k}=\left(k_{n}\right)_{n \geqslant 1}$, is not obviously implied by (2) but is implied by (3). It is however possible to establish (1) for particular $\mathbf{k}=\left(k_{n}\right)_{n \geqslant 1}$ for ergodic $T$ without recourse to (3) as the following Theorem demonstates. This suggests that (1) for ergodic $T$ is not equivalent to either (2) or (3).

THEOREM C. For a sequence of integers $\mathbf{k}=\left(k_{i}\right)_{i=1}^{\infty}$ suppose that the system of neighbourhoods $A_{n}=[1, n] \cap \mathrm{k}(n=1,2, \cdots)$ satisfies

$$
\left|A_{n} \Delta\left(h+A_{n}\right)\right|=o\left(\left|A_{n}\right|\right),
$$

for any $h$ in $\mathbf{N}$, where $\Delta$ denotes the symmetric difference of two sets, and the set $h+A_{n}$ denotes $\left\{h+k: k \in A_{n}\right\}$. Then (3) follows if for each set $A$ in the $\sigma$-algebra $\beta$ we have

$$
\lim _{N \rightarrow \infty} \frac{1}{N} \sum_{i=1}^{N} \mu\left(T^{-k_{i}} A \cap A\right)=\mu(A)^{2} .
$$

Proof: Recall that $L^{2}$ is a Hilbert space under the inner product $\langle f, g\rangle$ $=\int_{X} f \bar{g} d \mu$, where $\bar{g}$ is the complex conjugate of $g$. Let $U f(x)=f(T x)$. Then $\|U f\|=\|f\|$ because $T$ is measure preserving.

In the special case where for $A$ in $\beta$ we set $a=\chi_{A}$ (the characteristic function of $A$ ) the hypothesis (5) may be rewritten

$$
\lim _{N \rightarrow \infty} \frac{1}{N} \sum_{i=1}^{N}\left\langle U^{k_{i}} a, a\right\rangle=\langle a, 1\rangle\langle 1, a\rangle .
$$

By taking linear combinations of characteristic functions, this statement is also seen to remain true for simple functions $a$. For arbitrary $L^{2}$ functions $f$ given $\varepsilon>0$ we can 
find simple functions $a$ such that $\|f-a\|_{2} \leqslant \varepsilon$. Further by $\left(5^{\prime}\right)$ we can find a natural number $n=n(\varepsilon)$ such that if $N \geqslant n(\varepsilon)$ then

$$
\left|\frac{1}{N} \sum_{i=1}^{N}\left\langle U^{k_{i}} a, a\right\rangle-\langle a, 1\rangle\langle 1, a\rangle\right| \leqslant \varepsilon .
$$

Thus, also if $N \geqslant n(\varepsilon)$ then

$$
\begin{aligned}
\left|\frac{1}{N} \sum_{i=1}^{N}\left\langle U^{k_{i}} f, f\right\rangle-\langle f, 1\rangle\langle 1, f\rangle\right| \leqslant \mid & \frac{1}{N} \sum_{i=1}^{N}\left\langle U^{k_{i}} f, f\right\rangle-\frac{1}{N} \sum_{i=1}^{N}\left\langle U^{k_{i}} a, f\right\rangle \mid \\
& +\left|\frac{1}{N} \sum_{i=1}^{N}\left\langle U^{k_{i}} a, f\right\rangle-\frac{1}{N} \sum_{i=1}^{N}\left\langle U^{k_{i}} a, f\right\rangle\right| \\
& +\left|\sum_{i=1}^{N}\left\langle U^{k_{i}} a, a\right\rangle-\langle a, 1\rangle\langle 1, a\rangle\right| \\
& +|\langle a, 1\rangle\langle 1, a\rangle-\langle f, 1\rangle\langle 1, a\rangle| \\
& +|\langle f, 1\rangle\langle 1, a\rangle-\langle f, 1\rangle\langle 1, a\rangle| \\
\leqslant & \frac{1}{N} \sum_{i=1}^{N}\left|\left\langle U^{k_{i}}(f-a), f\right\rangle\right| \\
& +\frac{1}{N} \sum_{i=1}^{N}\left|\left\langle U^{k_{i}} a, f-a\right\rangle\right| \\
& +\varepsilon+|\langle f-a, 1\rangle\langle 1, a\rangle|+|\langle f, 1\rangle\langle 1, f-a\rangle| .
\end{aligned}
$$

Using the fact that $\|f\|=\langle f, f\rangle^{1 / 2}$ and Cauchy's inequality this is

$$
\leqslant \varepsilon\|f\|+\varepsilon(\|f\|+\varepsilon)+\varepsilon+\varepsilon(\|f\|+\varepsilon)+\varepsilon\|f\| .
$$

Thus we have shown that if $f \in L^{2}$ then

$$
\lim _{N \rightarrow \infty} \frac{1}{N} \sum_{i=1}^{N}\left\langle U^{k_{i}} f, f\right\rangle=\langle f, 1\rangle\langle 1, f\rangle
$$

Now $L^{2}=H \oplus H^{\perp}$ where $H=\bigcap_{n=1}^{\infty} U^{n} L^{2}(X, \beta, \mu)$ and note that if

$$
V=L^{2} \ominus U L^{2}=\left\{f \in L^{2}(X, \beta, \mu): f \perp L^{2}\left(X, T^{-1} \beta, \mu\right)\right\}
$$

where $T^{-1} \beta$ is the $\sigma$ algebra generated by $\left\{A=T^{-1} B: B \in \beta\right\}$, then

$$
H^{\perp}=\bigoplus_{n=0}^{\infty} U^{n} V
$$


Since the spaces are mutually orthogonal it is clear that for $f \in U^{i} V$ and $g \in U^{j} V$ with $i \neq j$ different we have

$$
\lim _{N \rightarrow \infty} \frac{1}{N} \sum_{i=1}^{N}\left\langle U^{k_{i}} f, g\right\rangle=0
$$

By taking linear combinations of such $f$ and $g$ and using approximation arguments in the $L^{2}(X, \beta, \mu)$ norm, we must have (7) for all $f$ and $g$ in $H^{\perp}$. Of course for $f$ in $H$ and $g$ in $H^{\perp}$ or vice versa then (7) is still true and we see that in order to prove Theorem $\mathrm{C}$ it suffices to show (7) assuming that $\int_{X} f(x) d \mu=0$. This is the only point at which we need to use the hypothesis on the sequence of integers $\mathbf{k}$. We first note that $U H=H$ and therefore $U$ is a unitary operator on $H$, that is, in particular it has an inverse there. Let $S(f)=\left\{U^{n} f: n \in \mathbf{Z}\right\}$, and let $Z(f)$ be the $\|$.$\| closure of$ the linear span of $S(f)$. Now by $(6)$ for arbitrary positive integers $\ell$

$$
\lim _{N \rightarrow \infty} \frac{1}{N} \sum_{i=1}^{N}\left\langle U^{k_{i}+\ell} f, U^{\ell} f\right\rangle=0 .
$$

Also by the hypothesis on the sequence $\mathbf{k}$ we have

$$
\sum_{i=1}^{N}\left\langle U^{k_{i}+\ell} f, U^{\ell} f\right\rangle=\sum_{i=1}^{N}\left\langle U^{k_{i}} f, U^{\ell} f\right\rangle+o(N)
$$

Taking linear combinations of the $U^{\ell} f$ and then taking limits completes the proof. $\quad$

\section{REFERENCES}

[1] R. Nair, 'On uniformly distributed sequences of integers and Poincaré recurrence', Indag. Math. (N.S.) 9 (1998), 55-63.

[2] R. Nair, 'On Hartman uniform distribution and measures on compact groups', in Harmonic Analysis and Hypergroups, (M. Anderson, A.I. Singh and K. Ross, Editors), Trends in Mathematics 3 (Birkhauser, Boston, M.A., 1998), pp. 59-75.

[3] R. Nair, 'On uniformly distributed sequences of integers and Poincaré recurrence II', Indag. Math. (N. S.) 9 (1998), 405-415.

[4] R. Nair and M. Weber, 'On random perturbations of some intersective sets', Indag. Math. (to appear). 